\title{
Effects of low intensity pulsed ultrasound stimulation on bone regeneration in rat parietal bone defect model
}

\author{
Kang Chen ${ }^{1 *}$, Jia Hao ${ }^{1}$, Kanako Noritake ${ }^{1}$, Yu Yamashita ${ }^{1}$, Shinji Kuroda ${ }^{1}$, Shohei Kasugai ${ }^{1,2}$ \\ ${ }^{1}$ Oral Implantology and Regenerative Dental Medicine, Tokyo Medical and Dental University, Tokyo, Japan; \\ *Corresponding Author: kangel.chan@gmail.com \\ ${ }^{2}$ Global Center of Excellence (G-COE), Tokyo Medical and Dental University, Tokyo, Japan
}

Received 20 January 2013; revised 19 February 2013; accepted 9 March 2013

\begin{abstract}
Purpose: Low intensity pulsed ultrasound stimulation (LIPUS) has been clinically applied to promote bone fracture healing in the orthopedic field. Thus, it is likely that LIPUS also stimulates bone regeneration in bone defects in the cranial-maxillofacial area. However, this has not been clearly proved. Furthermore, optimal time point and period of the application after the surgery has not been reported. The purpose of the present study was to evaluate the effect of LIPUS on bone regeneration in the rat parietal bone defects especially focusing on time and period of the application. Materials and Methods: Eighteen Wistar rats (14 weeks old) were divided into 6 groups: 5 experimental groups and a control group. Bone defect of $5 \mathrm{~mm}$ diameter was prepared on each side of the parietal bone and customized gelatin membranes were placed over the bone defects. LIPUS $\left(160 \mathrm{~mW} / \mathrm{cm}^{2}, 15\right.$ min/day) was applied to the defect area with an active transducer externally in the experimental groups according to the schedules of the applications: Group 1 (day 6 - 12), group 2 (day 13 19), group 3 (day 20 - 26), group 4 (day 6 - 19) and group 5 (day 6 - 26). All the animals were sacrificed at 28 days. The defects were analyzed with micro CT and then histologically. Results: In Group 1, new bone formation was significantly promoted and the newly-formed bone was thick and matured compared to the one of the control group. In other experimental groups there were tendencies of stimulation of new bone formation; however, they were not statistically significant. Discussion and Conclusion: The present study demonstrated that amount of new bone formation in the bone defect depended on the time and period of LIPUS appli-
\end{abstract}

cation. It has been suggested that application of LIPUS at an early healing period, the second week after the surgery, effectively accelerated new bone formation.

Keywords: LIPUS; Bone Regeneration; Bone Defect; Cranial-Maxillofacial

\section{INTRODUCTION}

Prosthetic treatment with dental implants has already been recognized as an effective modality for substituting lacking teeth and its long term prognosis is predictable. Notably, this modality needs bone to stabilize the implants; therefore, bone augmentation is often required in bone reduction cases [1]. Several bone augmentation techniques have been developed and clinically applied. However, the augmentation procedure for dental implant treatments often increases the total treatment period, which is obviously a burden on the patients.

It has been reported that application of low-intensity pulsed ultrasound stimulation (LIPUS) promotes fracture healing and LIPUS has been clinically applied in the orthopaedic field [2,3], where positive effects of LIPUS on fracture healing of long bone were clearly concluded to reduce the time frame of clinical and radiographic healing by $38 \%$ [4]; however, effects of LIPUS on bone regeneration of flat bones in the cranial-maxillofacial area have not been abundantly documented although promotion of reossification ratio in rat noncritical-sized parietal bone defects by LIPUS or by low magnitude and high frequency mechanical stimuli has been recently reported [5,6] and although some clinicians have already performed LIPUS for bone augmentations in this area. Effects of LIPUS on bone regeneration in the cranial-maxillofacial should be carefully evaluated in animal experiments prior to its clinical trials.

The purpose of the present study was to evaluate effects of LIPUS on bone regeneration in a parietal bone 
defect, especially focusing on time points and periods of the LIPUS application.

\section{MATERIALS AND METHODS}

\subsection{Animals}

Thirty six defects on the parietal bone of eighteen 14-week-old male Wistar rats weighing 330 - 350 g were assigned into 6 groups. One control group and five LIPUS application groups were divided based on the different treatment periods of LIPUS and 6 defects in each group were taken into account respectively. The animal experiment protocol was approved by the institutional committee for animal experiments of Tokyo Medical and Dental University.

\subsection{Surgical Procedure}

Anesthesia was achieved with a combination of Ketamine and Xylazine (40 and $5 \mathrm{mg} / \mathrm{kg}$, respectively). The scalps of rats were shaved and disinfected using iodine and $70 \%$ alcohol. A bowed incision was made coronally between the ears, and the skin and the connective tissue were exfoliated forward to the posterior end of the frontal bone. Subsequently, the periosteum was incised along the suture between the occipital and the parietal bones and elevated to expose the parietal bone surface. One full-thickness bone defect was created in each parietal bone by removing cranial disks with $5 \mathrm{~mm}$ in diameter carefully to avoid damage to the dura mater using a trephine bur constantly cooled with sterile saline (Figure 1(A)). And then a customized gelatin membrane, which was provided for guided bone regeneration by Nippi Co. Ltd., Tokyo, Japan [7], was placed to cover the defects of the both sides under the periosteum (Figure 1(B)). The periosteum and the skin were finally replaced with an absorbable and an unabsorbable suture $(4 \times 0$ polyglactin 910 and $4 \times 0$ silk, respectively) (Figure 1(C)). The day of surgery was considered as day 0 and the rats received standard laboratory food and water ad libitum for the
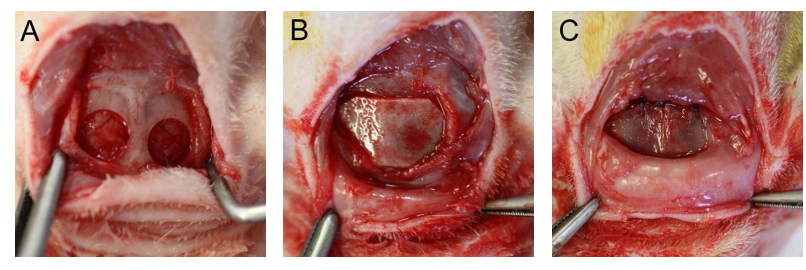

Figure 1. (A) One full-thickness bone defect was created in each parietal bone by removing cranial disks with $5 \mathrm{~mm}$ in diameter carefully to avoid damage to the dura mater using a trephine bur constantly cooled with sterile saline; (B) A customized gelatin membrane was placed to cover the defects of both sides under the periosteum; (C) The periosteum and the skin were finally replaced with an absorbable or an unabsorbable suture respectively. given period for each analysis.

\subsection{LIPUS Application}

A commercial ultrasound device (BR-Sonic, Ito Physiotherapy \& Rehabilitation Co., Ltd., Tokyo, Japan) was used to generate an intensity of $160 \mathrm{~mW} / \mathrm{cm}^{2}$ ultrasound $(5 \mathrm{MHz}, 100-\mathrm{Hz}$ pulse repetition frequency, $30 \%$ duty cycle).

Five different combinations of timing and repetition of LIPUS was scheduled for 5 groups, respectively, which was based on a unit of daily 15-minunte stimulation for six consecutive days followed by a no-LIPUS day. The rats in LIPUS application groups were wrapped with towel and fixed into a plastic slot which was used to restrict their excessive movements shown in Figure 2(A). The LIPUS transducer was fixed on the scalp to be positioned over the two defects with a gel for airtight contact between the device and the skin shown in Figure 2(B) and then the ultrasound was administered to the 5 groups according to the given schedule (Figure 3 ).

\subsection{Micro-CT}

All the animals were sacrificed at 28 days under excessive chloroform anesthesia. The defects with the surrounding cranial bone and soft tissue were removed and scanned by micro-CT (InspeXio; Shimadzu Science East Corporation, Tokyo, Japan) to obtain 3D microstructural information with a voxel size of $50 \mu \mathrm{m} / \mathrm{pixel}$. Threedimensional volume of the whole thickness radiopacity in $4.5 \mathrm{~mm}$ diameter at the center of each defect was rendered and calculated as new bone using Tri/3D-Bon software (RATOC System Engineering Co., Tokyo, Japan).
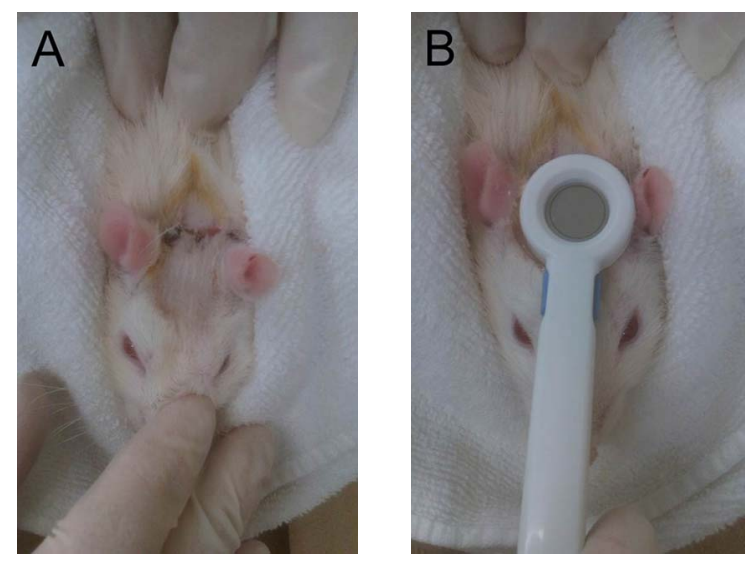

Figure 2. (A) The rats in LIPUS application groups were wrapped with towel and fixed into a plastic slot which was used to restrict their excessive movements; (B) The LIPUS transducer was fixed on the scalp to be positioned over the two defects with a gel for airtight contact between the device and the skin. 


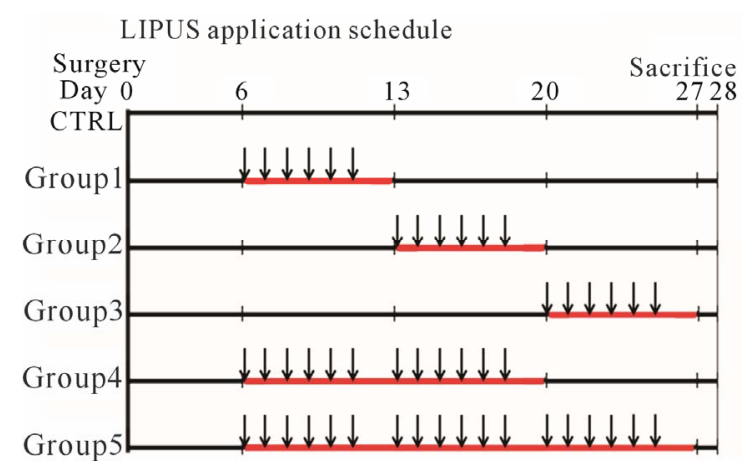

Figure 3. LIPUS application schedule showed as follows: After post-operative 5 days, the LIPUS intervention was commenced without daily basis anesthesia from day 6 to day 12 to assign as application group 1; from day 13 to day 19 as application group 2; from day 20 to day 26 as application group 3; from day 6 to day 12 and from day 13 to day 19 (two weeks) as application group 4; and from day 6 to day 12 and from day 13 to day 19 and from day 20 to day 16 (three weeks) as application group 5 . The red line indicated application period and the arrow indicated application times.

\subsection{Histological Analysis}

The specimens were fixed in 10\% neutralized formalin for two weeks, decalcified in formic acid for one month, dehydrated and finally embedded in paraffin. Approximate 5 - $\mu \mathrm{m}$-thickness coronal sections were prepared through the center of defects, stained with hematoxylin -eosin and observed under an optical microscope (Biozero, Keyence, Tokyo, Japan). The histological images were captured and stored in the computer. The newly formed bone area was measured with an image analyzer (Image J, National Institute of Health, USA) and expressed in percentage to the total defect area.

\subsection{Statistical Analysis}

Statistical analysis was performed by the statistic software PASW Statistics 18 for Windows. Data were first analyzed by one-way ANOVA. When this analysis suggested a significant difference between groups $(\mathrm{p}>$ 0.05 ), the data were further analyzed by Sheffe post hoc multiple comparison tests. $P$ values of less than 0.05 were considered to be statistically significant.

\section{RESULTS}

\subsection{Macroscopic Observation}

All the animals recovered well after surgery, and they were obedient, getting used to the daily treatment. And the soft tissue wounds healed uneventfully without infection except for general post-op inflammation over the experimental period. During the treatment, we observed small bubbles inside the ultrasound gel be- tween the transducer and the skin. These bubbles expanded and contracted into a visible size and then stabilized. Only one rat in the application group 4 showed a little relaxing symptom after the last-two-day treatment; however, it recovered soon. The body weight between the control and the application groups didn't show significant difference at the sacrifice.

\subsection{Radiological Observation}

Radiopacity in the defect was observed in both the untreated control group and the LIPUS intervention groups (Figure 4). The newly generated radiopacity that was especially close to the defect margin looked similar to that of the original bone, which might indicate that new bone had been more matured in the experimental models. Furthermore, compared to the control group, the LIPUS application groups showed larger amount of new bone: especially in group 1, thicker new mineralization occupied the whole defect area; and groups 1 and 2 showed more radiopacity than the control group, especially significant increase in group 1, which were calculated in the center area with $4.5 \mathrm{~mm}$ in diameter of the bone defect although the other application groups showed only slight increase in radiopacity (Figure 5).

\subsection{Histological Observation}

In the control group, a thin layer of new bone was formed; however, the defect closure with new bone was not completed because of invasion of fibrous tissue into the healing site.

In group 1, the whole defect was fulfilled with newly formed bone, which connected with the peripheral original bone so that the interface between the newly formed bone and the original bone was difficult to be identified as well as seen in the radiological assessment. Osteoid but irregular structure of immature bone rimmed by osteoblasts was observed in the central part of the defect. Numerous blood vessels existed inside the osteoid tissue. Active osteoblasts aligned along the edge of newly formed bone or on the periosteal side.

Percentage of the regenerated bone in the defect area of the control group was only $34.55 \% \pm 16.26 \%$, whereas those of groups 1 and 2 reached $77.53 \% \pm 16.17 \%$ and $69.89 \% \pm 14.42 \%$ respectively which exceeded signifycantly new bone regeneration in the untreated control group (Figures 6 and 7).

\section{DISCUSSION}

LIPUS began to be widely used clinically to treat fresh fractures in the early 1990s and non-unions or delayed unions in the late 1990s, although experimental studies have demonstrated that LIPUS enhances bone regenera- 

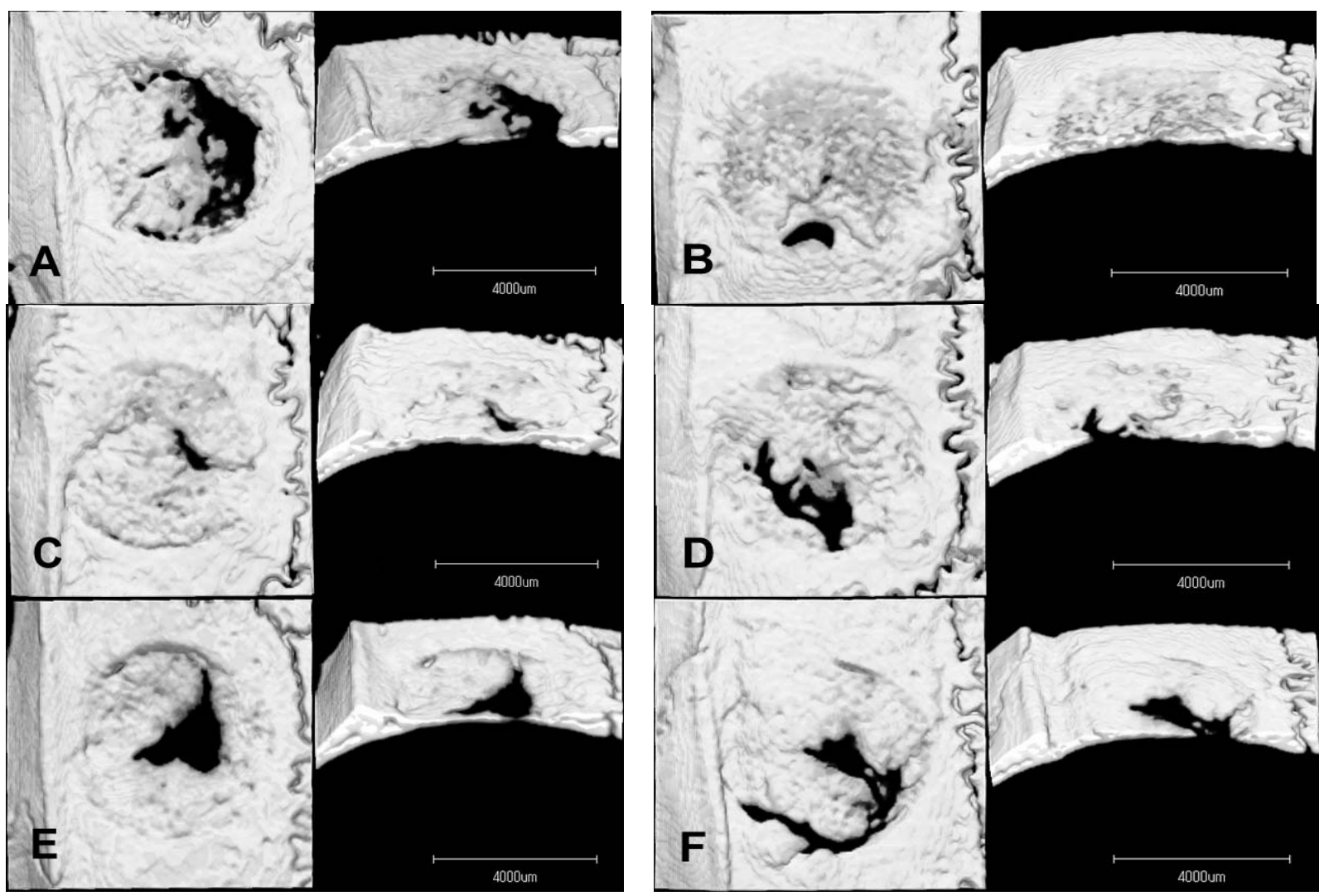

Figure 4. Micro CT images of the parietal defect at 4 weeks. Radiopacity in the defect was observed in both the untreated control group and the LIPUS intervention groups. The newly generated radiopacity that was especially close to the defect margin looked similar to that of the original bone, which might indicate that new bone had been more matured in the experimental models. Compared to the control group, the LIPUS application groups showed larger amount of new bone: Especially in group 1. Control (A); Group 1(B); Group 2(C); Group 3(D); Group 4(E); Group 5(F).

New Bone Volume $\left(\mathrm{mm}^{3}\right)$ in the Defect

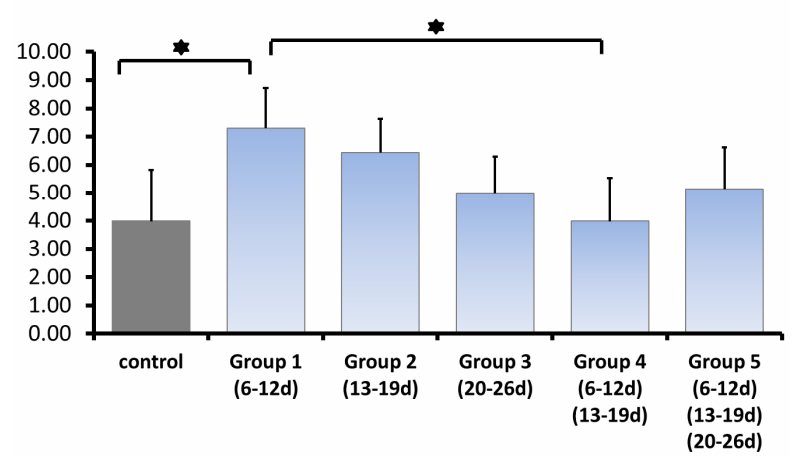

Figure 5. The newly generated radiopacity of each group: $3.98 \pm 1.84 \mathrm{~mm}^{3}$ in the control group; $7.30 \pm 1.43 \mathrm{~mm}^{3}$ in application group $1 ; 6.44 \pm 1.20 \mathrm{~mm}^{3}$ in application group 2; $4.99 \pm 1.30 \mathrm{~mm}^{3}$ in application group $3 ; 3.98 \pm 1.56 \mathrm{~mm}^{3}$ in application group $4 ; 5.13 \pm 1.50 \mathrm{~mm}^{3}$ in application group 5 , calculated in the center area with $4.5 \mathrm{~mm}$ in diameter of the bone defect. * showed statistically different $(\mathrm{P}<0.05)$.

tion during fracture healing and callus distraction quite recently [8-10]. Thus, it has been established that LIPUS stimulates bone regeneration in orthopedic field. How- ever, it was not clear that LIPUS stimulates bone regeneration in the maxillofacial area because regenerative process of a bone commonly generally follows its embryological process. Bones in the maxillofacial area are flat and formed by intramembranous ossification whereas most bones in other parts of the body are long bones and produced by endochondral ossification [11]. It has been recently reported that LIPUS also stimulates bone regeneration in calvaria bone defects in rats $[5,12]$. Furthermore, some dentists started to apply LIPUS clinically to the bone augmentation site in the maxillofacial area, such as sinus floor augmentation in Japan. However, optimal time points and periods of LIPUS application were not clear.

The present study demonstrated that amount of new bone formation in the bone defect depended on the time and period of LIPUS application. The intensity and frequency of LIPUS used in this study should be also taken into account as the factors influencing the results. However, in the present experimental conditions, it was sug gested that application of LIPUS at an early healing period, the second week after the surgery, effectively accelerated new bone formation. 

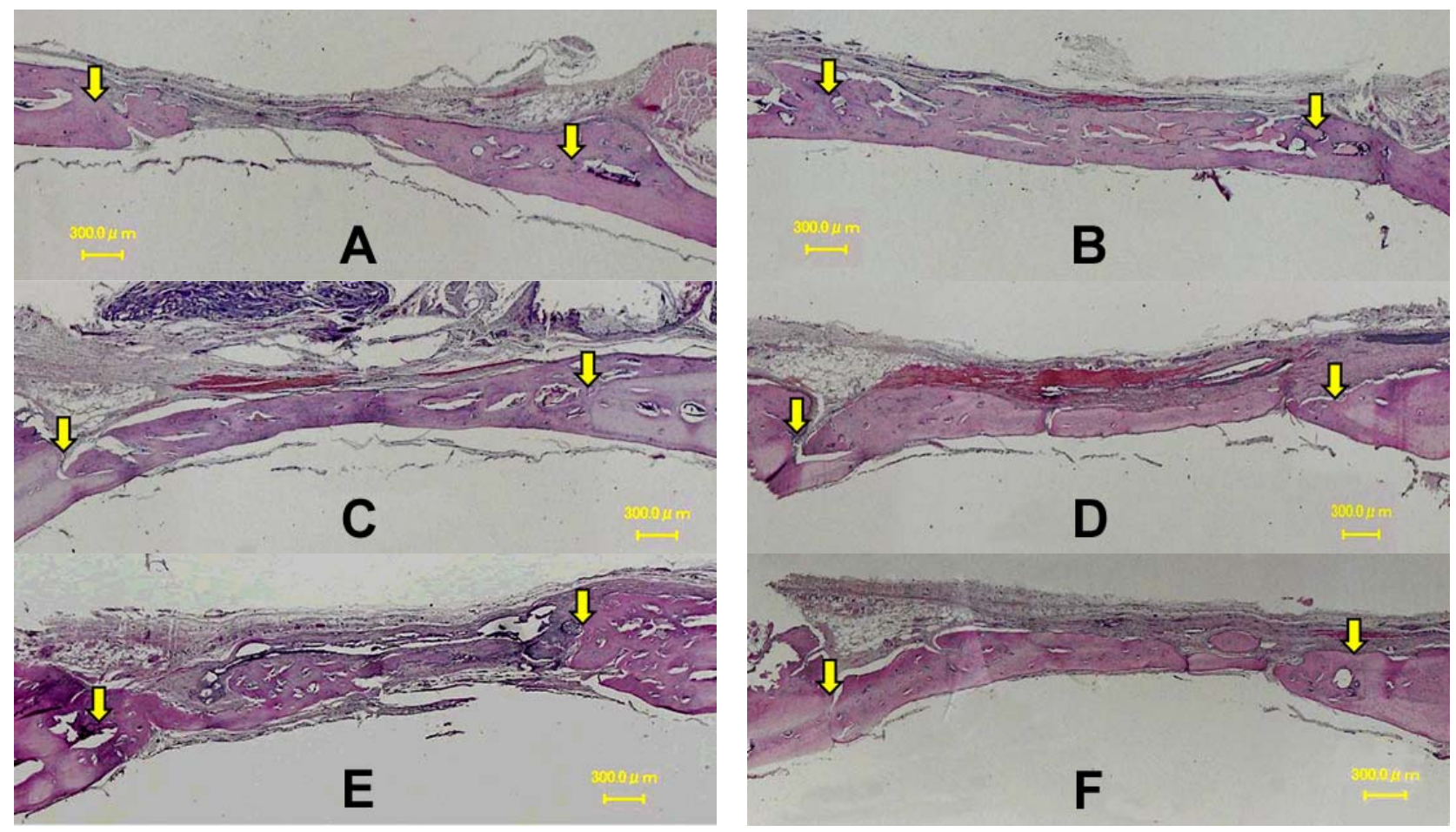

Figure 6. Histological images at 4 weeks. In the control group, a thin layer of new bone was formed; however, the defect closure with new bone was not completed because of invasion of fibrous tissue into the healing site. In group 1, the whole defect was fulfilled with newly formed bone, which connected with the peripheral original bone so that the interface between the newly formed bone and the original bone was difficult to be identified. Osteoid but irregular structure of immature bone rimmed by osteoblasts was observed in the central part of the defects. Control (A); Group 1(B); Group 2(C); Group 3(D); Group 4(E); Group 5(F).

Bone Fill Percentage \%

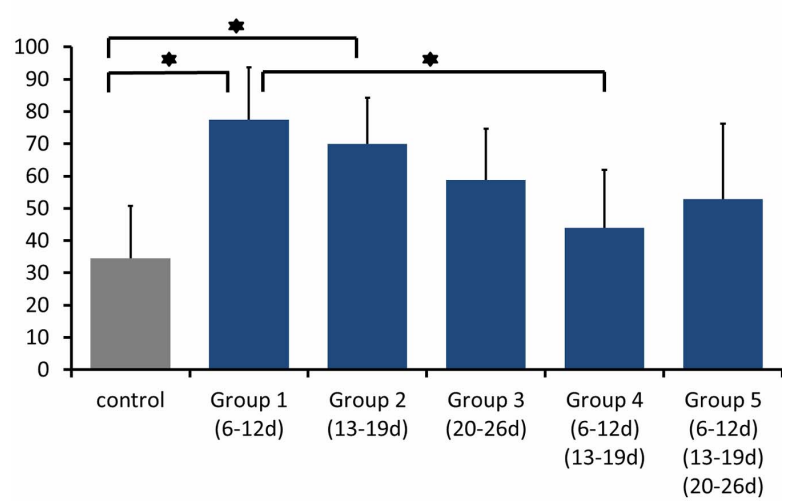

Figure 7. Percentage of the regenerated bone in the defect area of the control group was only $34.55 \% \pm 16.26 \%$, whereas those of groups 1 and 2 reached $77.53 \% \pm 16.17 \%$ and $69.89 \%$ $\pm 14.42 \%$ respectively which exceeded significantly new bone regeneration in the untreated control group. *showed statistically different $(\mathrm{P}<0.05)$

In the previous animal experiment LIPUS was applied from 24 hours after the surgery (Hasuike et al.). We considered that putting ultrasound gel and the transducer directly on unhealed surgical site was neither proper nor clinically practical although LIPUS application at this stage might stimulate bone regeneration [13]. Thus, we did not apply LIPUS from the day 1 to day 5 after the surgery and started the application at day 6 in the present study.

The defect was obviously filled with blood after surgical preparation, and then, coagulation process was activated producing fibrin and signal molecules, such as PDGF and TGF-beta from platelets [14]. These events were followed by early inflammatory response: blood vessel dilation with permeability increase resulting in cell filtrates [15]. At this stage swelling and pain are clinically evident, which is the early stage of inflammation, usually occurs within one week after surgical trauma. Then, granulation tissue formation accompanying extensive angiogenesis in the defect occurred at the next stage. The most effective time point of LIPUS application in the present study corresponded to this stage [16].

After vascularized primitive connective tissue is formed, bone spicules fuse with adjacent spicules to become trabeculae and woven bone, as a crucial step in the process of intramembranous ossification of the parietal bones. Ultrasound stimulation is considered to favor this stage, but afterwards, when the osteogenic differentiation occurs, ultrasound would induce potential negative effects due to temperature rise in previous studies $[17,18]$. That also might explain the bone volume decrease in group 4 of our present study. The slight positive effect in 
group 5 was probably related to effects of mechanical stimuli on the period of bone remodeling.

The general clinical formula of LIPUS application is $20 \mathrm{~min}$ per day, $1 \mathrm{MHz}$ sine waves repeating at $1 \mathrm{kHz}$, average intensity $30 \mathrm{~mW} / \mathrm{cm}^{2}$ and pulse width $200 \mu \mathrm{s}$. However, there is no consensus about the therapeutic LIPUS intensity. Lavandier and his collaborators have reported that LIPUS at $300 \mathrm{~mW} / \mathrm{cm}^{2}$ shows more bone regenerative effect than at $100 \mathrm{~mW} / \mathrm{cm}^{2}$ in a $3 \mathrm{~mm}$ noncritical size bone defects of the rat calvarium [19]. In the present study, considering the attenuation, the penetrative ability of LIPUS and the localization of the bone defect from the transducer, we used a setting of $5 \mathrm{MHz}$ frequency $160 \mathrm{~mW} / \mathrm{cm}^{2}$ intensity of LIPUS in $5 \mathrm{~mm}$ critical size bone defects of rat calvarium. This setting varied from the condition of stimulating bone regeneration in the rat calvaria bone defects, which has been recently reported [5,20], yet agreed to be the safe usage and dosage of ultrasound reported by Warden, S.J., et al. [21].

According to the literature, Utrasound therapy is reported to have two main relative effects, thermal and non-thermal, which are thought to be optimized through dosage modification. The intensity at which ultrasound is applied in regular clinical applications ranges from 0.1 to $1.0 \mathrm{~W} / \mathrm{cm}^{2}$. Some references stated that it required precaution when heating the skull if intensity of the ultrasound was more than $94 \mathrm{~mW} / \mathrm{cm}^{2}$ transcranially. Although the settings of the parameters in this present study effectively stimulated bone regeneration, the safety of each parameter should be clarified with more extensive studies.

In the present study we found that the application of LIPUS at the second week after surgery effectively stimulated bone regeneration. Although results in animal experiments do not always reflect the clinical situations in patients, it is likely that application of LIPUS at this particular stage after the surgery could also promote bone regeneration in patients. This LIPUS application protocol would be beneficial to patients because it reduces treatments and hospital visits. However, effectiveness and safety of this LIPUS application protocol should be clinically proven in the near future.

The effects of mechanical stress on cells including osteoblasts have been extensively studied in cell cultures. Possible signal molecules, which involved in the promoting effects of LIPUS in the present study, might be the followings: Prostaglandin E2, nitric oxide, vascular endothelial growth factors (VEGF) because enhancement of the production of these signal molecules by mechanical stress has been reported [22-24]. Although the precise mechanism of the present enhancement of bone regeneration is not clear, it is likely that LIPUS stimulated cellular migration, proliferation and differentiation which were controlled by these signal molecules $[25,26]$. It has been reported that LIPUS stimulated angiogenesis and the most effective time point of LIPUS application was at the second week when extensive angiogenesis generally occurs after the surgery $[27,28]$. Thus, promotion of angiogenesis by LIPUS seemed to contribute to the present enhancement of bone regeneration.

Conclusively, the present study demonstrated the beneficial effect of LIPUS application on bone regeneration in a rat parietal defect depending on time and period of the application and that the application of LIPUS at the second week for one week after the surgery was the most effective. These results indicated that time and period of LIPUS application should be considered clinically for promoting bone regeneration in the patient effectively, and the safety of LIPUS parameters should be further established.

\section{ACKNOWLEDGEMENTS}

We gratefully thank Dr. Warunee Pluemsakunthai and Dr. Lin Zayar for their help on applying daily LIPUS treatment.

\section{REFERENCES}

[1] Ustun, Y., et al. (2008) Effects of low-intensity pulsed ultrasound on dental implant osseointegration: A preliminary report. European Journal of Dentistry, 2, 254-262. doi:10.1080/17482960802566824

[2] Claes, L. and Willie, B. (2007) The enhancement of bone regeneration by ultrasound. Progress in Biophysics and Molecular Biology, 93, 384-398.

doi:10.1016/j.pbiomolbio.2006.07.021

[3] Hadjiargyrou, M., et al. (1998) Enhancement of fracture healing by low intensity ultrasound. Clinical Orthopaedics and Related Research, 355, S216-S229. doi:10.1097/00003086-199810001-00022

[4] Warden, S.J., et al. (2000) Acceleration of fresh fracture repair using the sonic accelerated fracture healing system (SAFHS): A review. Calcified Tissue International, 66, 157-163. doi: $10.1007 / \mathrm{s} 002230010031$

[5] Hasuike, A., et al. (2011) In vivo bone regenerative effect of low-intensity pulsed ultrasound in rat calvarial defects. Oral Surgery, Oral Medicine, Oral Pathology, Oral Radiology and Endodontology, 111, e12-e20. doi:10.1016/j.tripleo.2010.09.061

[6] Omar, H., et al. (2008) Effect of low magnitude and high frequency mechanical stimuli on defects healing in cranial bones. Journal of Oral and Maxillofacial Surgery, 66, 1104-1111. doi:10.1016/j.joms.2008.01.048

[7] Noritake, K. (2011) Development of a new barrier membrane for guided bone regeneration. Journal of Oral Tissue Engineering, 9, 53-63.

[8] Kinami, Y., Noda, T. and Ozaki, T. (2013) Efficacy of low-intensity pulsed ultrasound treatment for managed fresh diaphyseal fractures of the low extremity multicenter retrospective cohort study. Journal of Orthopaedic Science, 3. 
[9] Xie, L.K., et al. (2011) A preliminary study of the effect of low intensity pulsed ultrasound on new bone formation during mandibular distraction osteogenesis in rabbits. International Journal of Oral and Maxillofacial Surgery, 40, 730-736. doi:10.1016/j.ijom.2011.03.016

[10] Sakurakichi, K., et al. (2004) Effects of timing of lowintensity pulsed ultrasound on distraction osteogenesis. Journal of Orthopaedic Research, 22, 395-403. doi:10.1016/S0736-0266(03)00181-5

[11] Tobita, K., et al. (2011) Effect of low-intensity pulsed ultrasound stimulation on callus remodelling in a gaphealing model: Evaluation by bone morphometry using three-dimensional quantitative micro-CT. Journal of Bone and Joint Surgery, 93, 525-530. doi:10.1302/0301-620X.93B4.25449

[12] Schortinghuis, J., et al. (2004) Ultrasound to stimulate mandibular bone defect healing: A placebo-controlled single-blind study in rats. Journal of Oral and Maxillofacial Surgery, 62, 194-201. doi:10.1016/j.joms.2003.06.006

[13] Leung, M.C., Ng, G.Y. and Yip, K.K. (2004) Effect of ultrasound on acute inflammation of transected medial collateral ligaments. Archives of Physical Medicine and Rehabilitation, 85, 963-966. doi:10.1016/j.apmr.2003.07.018

[14] Resnick, N., et al. (1993) Platelet-derived growth factor B chain promoter contains a cis-acting fluid shear-stressresponsive element. Proceedings of the National Academy of Sciences of USA, 90, 4591-4595. doi:10.1073/pnas.90.10.4591

[15] Rawool, N.M., et al. (2003) Power doppler assessment of vascular changes during fracture treatment with lowintensity ultrasound. Journal of Ultrasound in Medicine, 22, 145-153.

[16] Azuma, Y., et al. (2001) Low-intensity pulsed ultrasound accelerates rat femoral fracture healing by acting on the various cellular reactions in the fracture callus. Journal of Bone and Mineral Research, 16, 671-680. doi:10.1359/jbmr.2001.16.4.671

[17] Yang, K.H., et al. (1996) Exposure to low-intensity ultrasound increases aggrecan gene expression in a rat femur fracture model. Journal of Orthopaedic Research, 14, 802-809. doi:10.1002/jor.1100140518

[18] Payton, O.D., Lamb, R.L. and Kasey, M.E. (1975) Effects of therapeutic ultrasound on bone marrow in dogs. Physical Therapy, 55, 20-27.

[19] Lavandier, B., Gleizal, A. and Bera, J.C. (2009) Experimental assessment of calvarial bone defect reossification stimulation using low-intensity pulsed ultrasound. Ultrasound in Medicine and Biology, 35, 585-594. doi:10.1016/j.ultrasmedbio.2008.10.002

[20] Duarte, L.R. (1983) The stimulation of bone growth by ultrasound. Archives of Orthopaedic and Trauma Surgery, 101, 153-159. doi:10.1007/BF00436764

[21] Warden, S.J. and McMeeken, J.M. (2002) Ultrasound usage and dosage in sports physiotherapy. Ultrasound in Medicine and Biology, 28, 1075-1080. doi:10.1016/S0301-5629(02)00552-5

[22] Reher, P., et al. (2002) Ultrasound stimulates nitric oxide and prostaglandin E2 production by human osteoblasts. Bone, 31, 236-241. doi:10.1016/S8756-3282(02)00789-5

[23] Reher, P., et al. (1999) Effect of ultrasound on the production of IL-8, basic FGF and VEGF. Cytokine, 11, 416-423. doi:10.1006/cyto.1998.0444

[24] Forwood, M.R. (1996) Inducible cyclo-oxygenase (COX-2) mediates the induction of bone formation by mechanical loading in vivo. Journal of Bone and Mineral Research, 11, 1688-1693. doi:10.1002/jbmr.5650111112

[25] Leung, K.S., et al. (2004) Low intensity pulsed ultrasound stimulates osteogenic activity of human periosteal cells. Clinical Orthopaedics and Related Research, 1, 253-259. doi:10.1097/00003086-200401000-00044

[26] Naruse, K., et al. (2010) Prolonged endochondral bone healing in senescence is shortened by low-intensity pulsed ultrasound in a manner dependent on COX-2. Ultrasound in Medicine and Biology, 36, 1098-1108. doi:10.1016/j.ultrasmedbio.2010.04.011

[27] Ramli, R., et al. (2009) The effect of ultrasound on angiogenesis: An in vivo study using the chick chorioallantoic membrane. International Journal of Oral and Maxillofacial Implants, 24, 591-596.

[28] McKenzie, J.A. and Silva, M.J. (2011) Comparing histological, vascular and molecular responses associated with woven and lamellar bone formation induced by mechanical loading in the rat ulna. Bone, 48, 250-258. doi:10.1016/j.bone.2010.09.005 